PROCEEDINGS OF THE

AMERICAN MATHEMATICAL SOCIETY

Volume 125, Number 6, June 1997, Pages 1689-1691

S 0002-9939(97)03980-4

\title{
INVARIANT SUBSPACES FOR POLYNOMIALLY HYPONORMAL OPERATORS
}

\author{
BEBE PRUNARU
}

(Communicated by Palle E. T. Jorgensen)

\begin{abstract}
We show that if $T$ is a bounded operator on a Hilbert space such that $p(T)^{*} p(T)-p(T) p(T)^{*} \geq 0$ for every polynomial $p$, then $T$ has a nontrivial invariant subspace.
\end{abstract}

Let $H$ be a complex separable Hilbert space, and let $L(H)$ denote the algebra of all bounded linear operators on $H$. An operator $T \in L(H)$ is hyponormal if $T^{*} T-T T^{*} \geq 0$ and polynomially hyponormal if $p(T)$ is hyponormal for every analytic polynomial $p \in C[z]$. Recall also that an operator $S \in L(H)$ is subnormal if it is the restriction of a normal operator to an invariant subspace. One can easily show that every subnormal operator is polynomially hyponormal. The question whether the converse is true had been open for a long time but recently, R. Curto and $\mathrm{M}$. Putinar $[\mathrm{CuP}]$ answered it negatively.

As we shall see below, polynomially hyponormal operators and subnormal operators still share some nice properties, especially when we look at the lattice of their invariant subspaces. Recall that a subalgebra $A \subset L(H)$ is said to be reflexive if $A=\operatorname{AlgLatA}$, where $\operatorname{AlgLat} A=\{T \in L(H): \operatorname{Lat} A \subset \operatorname{Lat} T\}$. An operator $T \in L(H)$ is said to be reflexive if the weakly closed subalgebra generated by $T$ in $L(H)$ is reflexive. The main result of this paper is the following theorem, which gives an affirmative answer to a question raised in [CuP, Problem 4.1]:

Theorem 1. Every polynomially hyponormal operator $T \in L(H)$ is reflexive.

For the case when $T$ is subnormal, this theorem has been proved by R. Olin and J. Thomson (cf. [OT]). An immediate consequence of Theorem 1 is the following.

Corollary 1. Every polynomially hyponormal operator has a nontrivial invariant subspace.

For subnormal operators, the existence of invariant subspaces was proved by S. Brown in [Br1]. Also, a large class of hyponormal operators have invariant subspaces (cf. [Br2]). The proof of Theorem 1 is essentially based on some deep results from the theory of dual algebras. A dual algebra $A \in L(H)$ (i.e. a weak* closed unital subalgebra of $L(H))$ is said to have property $\left(A_{1}(r)\right)$ for some $r \geq 1$ if for every weak* continuous linear functional $\phi$ on $A$, and for every $s>r$, there exist vectors $x$ and $y$ in $H$ such that $\phi(T)=(T x, y)$ for every $T \in A$ and moreover, $\|x\| \cdot\|y\| \leq s\|\phi\|$

Received by the editors October 30, 1995.

1991 Mathematics Subject Classification. Primary 47A15, 47B20; Secondary 47D25, 47D27.

Key words and phrases. Hyponormal operators, dual algebras, invariant subspaces.

(C) 1997 American Mathematical Society 
(see [BFP, p. 7]). For any $T \in L(H)$ let $A_{T}$ denote the dual algebra generated by $T$ in $L(H)$. Suppose that $T \in L(H)$ is such that $A_{T}$ is isometrically isomorphic and weak ${ }^{*}$ homeomorphic with $H^{\infty}(D)$, the algebra of all bounded analytic functions in the unit disc $D$. In this case, it was proved independently by H. Bercovici [B] and B. Chevreau $[\mathrm{Ch}]$ that $A_{T}$ has property $\left(A_{1}(r)\right)$ for some universal constant $r \geq 1$ (Bercovici gets the best value $r=1$ ). Moreover, S. Brown and B. Chevreau proved that every such algebra is reflexive (cf. [BrCh]).

On the other hand, G. Cassier showed that if for a given $T \in L(H)$, the dual algebra $A_{T}$ is uniform, then it can be written as $A_{T}=\bigoplus_{i \geq 0} A_{i}$, where $A_{0}$ is an abelian von Neumann algebra and for every $i \geq 1, A_{i}$ is isometrically isomorphic and weak* homeomorphic with $H^{\infty}(D)$ (cf. [Cas]). A similar decomposition for dual Banach algebras is given in [Pr2]. Recall now that by the von Neumann double commutant theorem, every von Neumann algebra is reflexive. Moreover, if it is abelian, then obviously it also has property $\left(A_{1}(1)\right)$. Now, taking direct sums of reflexive algebras with property $\left(A_{1}(1)\right)$, we are still in the same class (cf. [BFP, p. 11]). As a consequence of these results, we have the following theorem, that will be very useful in the sequel.

Theorem 2. Let $T \in L(H)$ be an operator such that $A_{T}$ is a uniform algebra. Then $A_{T}$ has property $\left(A_{1}(1)\right)$ and is reflexive.

In order to apply the above theorem, we need one more result.

Proposition 1. Let $T \in L(H)$ be a polynomially hyponormal operator. Then every operator $S$ in the weakly closed algebra $W_{T}$ generated by $T$ in $L(H)$ is hyponormal.

Proof. Let us first observe that the weak and the strong-* closure of the linear space $\{p(T): p \in C[z]\}$ coincide (see [D, p. 40]). Therefore, given $S \in W_{T}$, there exists a net $\left\{p_{i}(T)\right\}$ such that $\left\|p_{i}(T) x-S x\right\|+\left\|p_{i}(T)^{*} x-S^{*} x\right\| \rightarrow 0$ for every $x \in H$. Therefore $\left\|S^{*} x\right\|=\lim \left\|p_{i}(T)^{*} x\right\| \leq \lim \left\|p_{i}(T) x\right\|=\|S x\|$ for every $x \in H$, hence $S$ is a hyponormal operator.

We are now able to prove the following structure theorem for the dual algebra generated by a polynomially hyponormal operator.

Theorem 3. If $T \in L(H)$ is a polynomially hyponormal operator, then the dual algebra $A_{T}$ generated by $T$ in $L(H)$ has property $\left(A_{1}(1)\right)$ and is reflexive.

Proof. Let us recall that the spectral radius of a hyponormal operator equals its norm (cf. [Co, p. 47]). Therefore, by Proposition 1, the dual algebra $A_{T}$ is uniform, and now a simple application of Theorem 2 yields the result.

In the case of a subnormal operator, the property $\left(A_{1}(r)\right)$ for $A_{T}$ was proved in [OT]. Subsequently, H. Bercovici and J. Conway showed that $r=1$ (cf. [BCo]).

Proof of Theorem 1. Follows easily from Theorem 3 and the fact that a dual algebra with property $\left(A_{1}(1)\right)$ is also weakly closed (cf. [BFP, p. 11]).

We close with a few remarks. Suppose that $T \in L(H)$ is a polynomially hyponormal operator without nonzero normal summand. Then the above mentioned decomposition for uniform dual algebras implies that $A_{T}$ is isometric and weak* homeomorphic with the algebra of bounded analytic functions on a planar open set $G$. Moreover, as shown in [Cas], the components of $G$ are simply connected. This shows that polynomially hyponormal operators have a very rich functional calculus. 
Suppose now that $T \in L(H)$ is such that $f(T)$ is hyponormal for every rational function $f$ with poles outside $\sigma(T)$. Then one can see that $\sigma(T)$ is a spectral set for $T$, in other words $T$ is a von Neumann operator. For such operators Theorem 3 has already been proved in $[\mathrm{CoDu}]$ and $[\mathrm{Pr} 1]$. In this context, the following question seems natural. Is there any polynomially hyponormal operator whose spectrum is not a spectral set? Since any subnormal operator is a von Neumann operator, an affirmative answer to this question would provide another proof of the existence of polynomially hyponormal operators which are not subnormal.

\section{REFERENCES}

[B] H. Bercovici, Factorization theorems and the structure of operators on Hilbert space, Annals of Math. 128 (1988), 399-413. MR 89i:47032

[BCo] H. Bercovici and J. B. Conway, A note on the algebra generated by a subnormal operator, Operator Theory: Adv. Appl. 32, 53-56. MR 90a:47058

[BFP] H. Bercovici, C. Foias and C. Pearcy, Dual Algebras with Applications to Invariant Subspaces and Dilation Theory, C.B.M.S. Regional Conf. Ser. in Math. no. 56, Amer. Math. Soc., Providence, R.I., 1985. MR 87g:47091

[Br1] S. Brown, Some invariant subspaces for subnormal operators, Integral Equations and Operator Theory 1 (1978), 310-333. MR 80c:47007

[Br2] , Hyponormal operators with thick spectrum have invariant subspaces, Annals of Math. 125 (1987), 93-103. MR 88c:47010

[BrCh] S. Brown and B. Chevreau, Toute contraction a calcul fonctionnel isometrique est reflexive, C. R. Acad. Sci. Paris, Serie I 307 (1988), 185-188. MR 89i:47007

[Cas] G. Cassier, Sur la structure d'algebres duales uniformes d'operateurs sur l'espace de Hilbert, C. R. Acad. Sci. Paris, Serie I 309 (1989), 479-482. MR 91f:47059

[Ch] B. Chevreau, Sur les contractions a calcul fonctionnel isometrique, II, J. Operator Theory 20 (1988), 269-293. MR 90f:47021

[Co] J. B. Conway, The Theory of Subnormal Operators, Amer. Math. Soc., Providence, RI, 1991. MR 92h: 47026

[CoDu] J. B. Conway and J. Dudziak, Von Neumann operators are reflexive, J. Reine Angew. Math. 408 (1990), 34-56. MR 91f:47003

[CuP] R. Curto and M. Putinar, Nearly subnormal operators and moment problems, J. Funct. Analysis 115 (1993), 480-497. MR 95d:47024

[D] J. Dixmier, Les Algebres d'Operateurs dans l'Espace Hilbertien, Gauthier-Villars, Paris, 1957. MR 20:1234

[OT] R. Olin and J. Thomson, Algebras of subnormal operators, J. Funct. Analysis 37 (1980), 271-301. MR 82a:47024

[Pr1] B. Prunaru, Von Neumann operators are reflexive, Integral Equations and Operator Theory 14 (1991), 609-612. MR 92h:47008

[Pr2] _ A structure theorem for singly generated dual uniform algebras, Indiana Univ. Math. J. 43 (1994), 729-736. MR 96e:47048

Department of Mathematics, Indiana University, Bloomington, Indiana 47405

Institute of Mathematics, Romanian Academy, P. O. Box 1-764, 70700 Bucharest, ROMANIA 